\title{
The Design of PROFIBUS-DP HUB based on FPGA
}

\author{
Jiao Ping \\ Lab. of Networked Control Systems \\ Shenyang Institute of Automation (SIA), Chinese \\ Academy of Sciences China, Shenyang \\ jiaoping@sia.cn
}

\author{
Zhou Tong \\ Lab. of Networked Control Systems \\ Shenyang Institute of Automation (SIA), Chinese \\ Academy of Sciences China, Shenyang \\ tzhou@sia.cn
}

\begin{abstract}
This paper analyzes the PROFIBUS-DP and physical layer signals states, DP time series and other characteristics. In the past, PROFIBUS-DP HUB common problems of poor universal apply, shape communication is more time-consuming, required message analysis, Drop frame etc, this paper propose a new design of PROFIBUS-DP HUB which based on FPGA. The design contain a detector which used to monitor the physical layer signal state of the DP bus, use of FPGA synthesis logic signal, could control the direction of data flow in the physical layer, and Unrelated with the upper layer protocol, without message analysis, compliance the protocol which used the standard RS-485, and baudrate-adaptive.
\end{abstract}

Keywords- PROFIBUS-DP; HUB; Baudrate-adaptive;

\section{INTRODUCTION}

PROFIBUS (Process Field Bus) is a standard for field bus communication in automation technology. The success of PROFIBUS is equally due to its advanced technology and the successful activities of the organization, which was founded to represent the interests of manufacturers and users[1].

Along with the continuous development of PROFIBUS, ROFIBUS-DP HUB devices widely used in the PROFIBUS DP networks, which could be convenient field installation, increase the transmission distance and the number of slave station, changing the PROFIBUS DP topology of the network. Finally, improved the system security, reliability, and upgraded convenience.

This paper propose a new design of PROFIBUS-DP HUB which based on FPGA, for the PROFIBUS-DP HUB common problems of poor universal apply, shape communication is more time-consuming, required message analysis, Drop frame etc. The design contain a detector which used to monitor the physical layer signal state of the DP bus, use of FPGA synthesis logic signal, could control the direction of data flow in the physical layer, and Unrelated with the upper layer protocol, without message analysis, compliance the protocol which used the standard RS-485, and baudrate-adaptive.

\section{PROFIBUS-DP}

PROFIBUS-DP uses the ISO/OSI model, Layer 1(Physical Layer, PHY), Layer 2 (Data Link Layer, FDL) and Layer 7 (Application Layer). The Physical Layer is specified the standard EIA RS-485, and the traffic rate is from $9.6 \mathrm{kbps}$ to $12 \mathrm{Mbps}[2,3,6]$.

IEC 1158-2 specified the station number of PROFIBUS-DP is 126 (station address from 0 to 125 contain master and slave station) [4]. In the one segment of PROFIBUS-DP, the maximum station is 32. DP HUB or DP Repeater device requires the use, if want to connect more stations or change the network topology. As figure shown, DP HUB or DP Repeater divided the DP network into many subnet segments [7]. Each interface of DP HUB is a Repeater, which can independently drive a PROFIBUS subnet segment, and connect up to 31 stations.

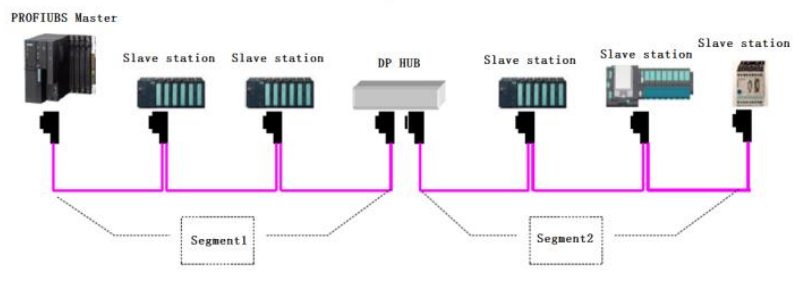

Figure 1. Network Topology of PROFIBUS-DP

\section{A. Timing of PROFIBUS-DP}

PROFIBUS-DP specified the frame structure and the bus timing to ensure the frame can be correct transmitted on the bus, and defined some parameters to ensure the interval transmitted time, delay time, and the maximum allowable waiting time. The main parameters were shown as follow [6].

- $t_{\text {BIT }}$ : The Bit Time $t_{\text {BIT }}$ is the time which elapses during the transmission of one bit. It is equivalent to the reciprocal value of the transmission rate:

$\mathrm{t}_{\mathrm{BIT}}=1 /$ Transmission Rate in bit/s.

- TSYN: The Synchronization Time: TSYN = 33 bit;

- $\quad \min$ TSDR, max TSDR: Minimum Station Delay of Responders and Maximum Station Delay of Responders (stations which acknowledge or respond):

$$
\begin{aligned}
& \min \mathrm{T}_{\mathrm{SDR}}=\min \mathrm{t}_{\mathrm{SDR}} / \mathrm{t}_{\mathrm{BIT}} ; \\
& \max \mathrm{T}_{\mathrm{SDR}}=\max \mathrm{t}_{\mathrm{SDR}} / \mathrm{t}_{\mathrm{BIT}} ;
\end{aligned}
$$

- TQUI: During this Quiet Time TQUI transmission and receipt of frames shall be disabled. This shall also be taken into account when using self-controlled 
repeaters, whose switching time shall be taken into consideration:

$$
\mathrm{T}_{\mathrm{QUI}}<\min \mathrm{T}_{\mathrm{SDR}}
$$

- Idle: the last bit of the received frame to the next frame start bit bus idle time, the response time of a request frame from the station Minimum:

$$
\min \mathrm{T}_{\mathrm{SDR}}=11 \mathrm{t}_{\mathrm{BIT}}
$$

\section{B. $D P H U B$}

PROFIBUS-DP used master and slave communication protocol and token-ring between the master stations, to avoid more than one station in the network to send frames at the same time, ensure the correctness communication. The basic function of DP HUB or other Repeater devices: When any one port receive message from DP bus, the other ports should be timeliness and correct forwarded out the message.

DP HUB in order to ensure the data forwarding accurately and, which should be know the frame start and stop bit for each frame. By monitoring the change of DP bus state in the Physical Layer can help to judge whether the port received a start bit data. It's relatively difficult judge the stop bit of message frame, at present, there are mainly two kinds of algorithms: The first one required message analysis, then read the content information of DP frame length byte, which can accurately calculate the frame stop bit position. Another one needed not message analysis, only by processing the logic signal in the physical layer signal, unrelated with the upper layer protocol.

\section{1) message analysis}

There are four frame types in the PROFIBUS-DP Data Link Layer as following, including : fixed-length data fields and no data frame (SD1), Variable length data (SD2), Fixed length data (SD3), the token frame (SD4), Brief acknowledgement frame (SC).

The master baud rate is should be know first before message analysis. PROFIBUS-DP supports 10 kinds of baud rate, according to the order from low to high (vice versa), with the baud rate to communicate that so-called "exhaustive". If the stipulated time or retry is not matching the correct message frame, then use the next baud rate, until a successful match. With the right baud rate can finish the message analysis, read the content information of length byte, which can help to accurately calculate the stop bit of the frame.

The algorithm advantages is able to accurately calculate the stop bit of the frame, the disadvantage is complex algorithm, shape communication is more time-consuming, which need to try $5 * 10-1$ times in theory, and poor universal application.

\section{2) Non message analysis}

Non message analysis, need not match the right baud rate, and do not finish message analysis, which forwarding the received frame, only by analysis logic signal of DP signal in the physical layer. That is: when any port of DP HUB bus state is low level signal (the bus's idle state is high level), the other port is arranged to transmit state, and bit by bit forwarding. When the low level change into high level, the other port should be receive state, and wait to forward out the receive frame.

The method of Non message analysis is easy to implement, without detecting stop bit of frame. The disadvantage is the low level signal interfere the other port, disrupt the right state and broadcast the error to the other ports, even paralysis the whole bus network, when a port is receiving state.

\section{Analysis and Design}

This paper proposed a new design of DP HUB, as shown in Figure 2. Bus transceiver processed the DP bus signal receive and transmit. The detector processed DP original A, B level signal, the logical result is Q_S. The FPGA is used to comprehensive and analysis the logic signal, which could control the direction of data flow in the physical layer, Unrelated with the upper layer protocol, without DP's packet analysis, compliance with the standard RS-485, and baudrate-adaptive.

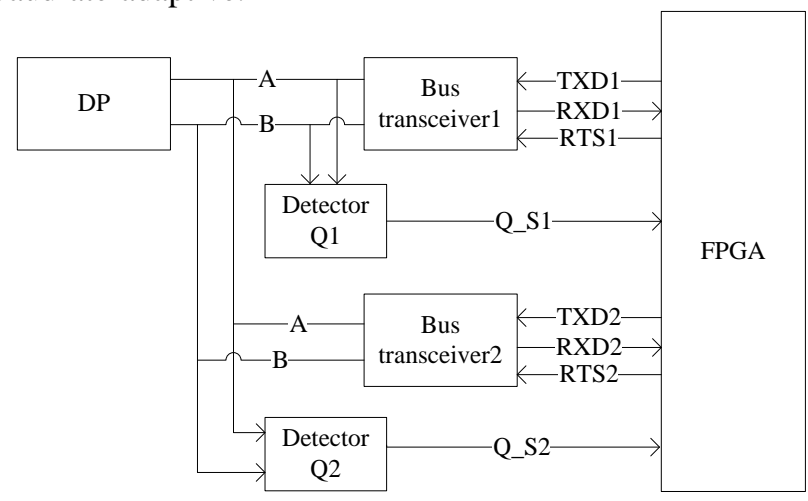

Figure 2. The scheme of PROFIBUS-DP HUB

\section{A. State machine}

As shown in Figure 3, there are three states of DP HUB: S0 wait to receive state; $\mathrm{S} 1$ receive and forward state; S2 ended forward state.

S0: After power-on reset, bus transceiver of each channel are set to receive state, monitoring start bit of frame at the same time.

S1: At some point detect start bit of frame in any channel, the other channels transceiver are set to transmit state, and forwards the received frame from DP.

S2: At some point detect ended bit of frame in any channel, each channel of transceiver are set to receive state, and system changed into the SO state.

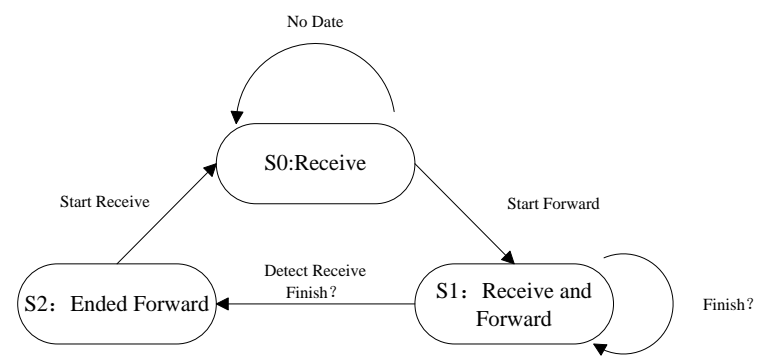

Figure 3. The state machine of DP HUB 


\section{B. Detector Design}

Physical Layer of the PROFIBUS Specification NRZ (Non Return to Zero) coded data is transmitted via a twisted cable. A binary " 1 " is represented by a constant positive differential voltage between A and B of the bus, a binary "0" by a constant negative differential voltage. As shown in Figure 4, when VA - VB> $200 \mathrm{mV}$ (bus is idle VA is about $3 \mathrm{~V}, \mathrm{VB}$ is about $2 \mathrm{~V}$ ) the RXD of bus transceiver output logic high level. When the bus is busying, the RXD still output logic high level, when VA - VB $>2 \mathrm{~V}$ (VA, VB maximum is about $4 \mathrm{~V}$, the minimum is about $1 \mathrm{~V})$.

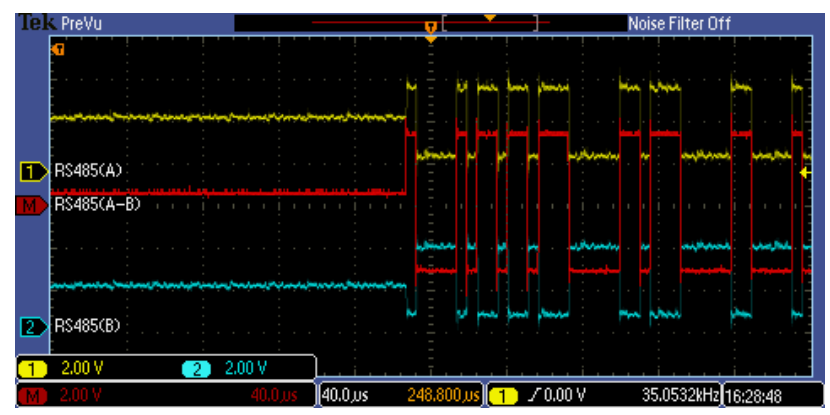

Figure 4. Idle time state of PROFIBUS-DP

In order to distinguish bus idle and work state of Profibus DP, and ensure the frame forwarding accurately, DP HUB need to detect the start bit of frame. This paper designed a detector, which used to be detect Vamp amplitude signal and process logic signal, also meet the signal delay and large signal response. The figure 5 is the schematic diagram of detector.

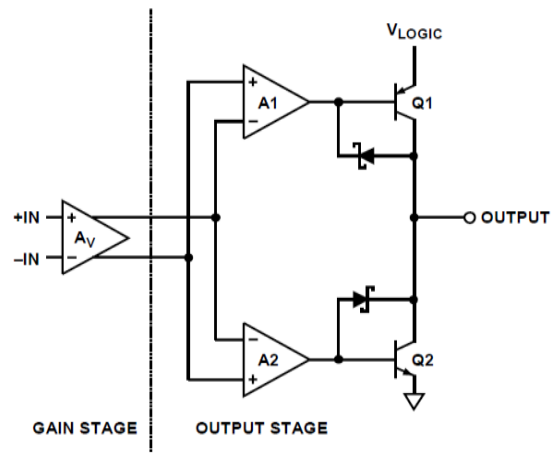

Figure 5. Simplified Schematic Diagram of Detector

The input signals of detector are VA and VB, $\mathrm{f}($ Vamp $)=\mathrm{f}(\mathrm{A})-\mathrm{f}(\mathrm{B})$ amplitude function $\mathrm{f}$ (Vamp) signal is arithmetic difference of $\mathrm{f}(\mathrm{A})$ and $\mathrm{f}(\mathrm{B})$, the result amplitude corresponding two state of $\mathrm{f}$ (Diff) function, as the equation(4) shown. Equation (5), $\mathrm{Q}_{\mathrm{S}}=\left(\frac{1}{\mathrm{f}(\text { Diff })}\right) \mid \mathrm{f}(\mathrm{RXD})$ means inverted $\mathrm{f}(\mathrm{Diff})$ signal and Logic or RXD signal that received by the transceiver, QS signal is the output signal of the detector for changing the state of the DP bus, as shown in equation(6), including the bus idle state into the working state from the "1 $\rightarrow 0$ ", and the working state into the idle state of "0 -> 1 ".

$$
\begin{aligned}
& \mathrm{f}(\mathrm{A})=\mathrm{f}(\text { VA }) \\
& \mathrm{f}(\mathrm{B})=\mathrm{f}(\text { VB }) \\
& \mathrm{f}(\text { Vamp })=\mathrm{f}(\mathrm{A})-\mathrm{f}(\mathrm{B}) \\
& \mathrm{f}(\text { Diff })=\left\{\begin{array}{l}
1 \text { Vamp }>2 \\
0 \text { Vamp }<2
\end{array}\right. \\
& \mathrm{Q}_{\mathrm{S}}=\left(\frac{1}{\mathrm{f}(\text { Diff })}\right) \mid \mathrm{f}(\mathrm{RXD}) \\
& \mathrm{Qs}_{\mathrm{s}}= \begin{cases}1 & \text { DP idle } \\
0 & \text { DP busy }\end{cases}
\end{aligned}
$$

As Figure 6 and 7 shown, the voltage amplitude and transmission delay of the input signal, the minimum value is less than $4 \mathrm{~ns}$, the rising and falling output signal settling time, respectively $35 \mathrm{~ns}$ and $45 \mathrm{~ns}$, when the voltage amplitude is $1.5 \mathrm{v}$ of input signals, which satisfy the maximum baud rate of DP.

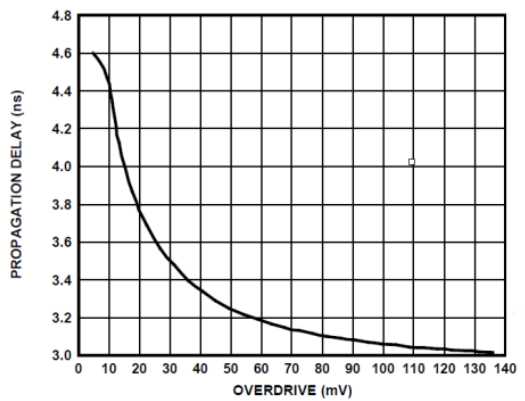

Figure 6. Propagation Delay vs Input Overdrive

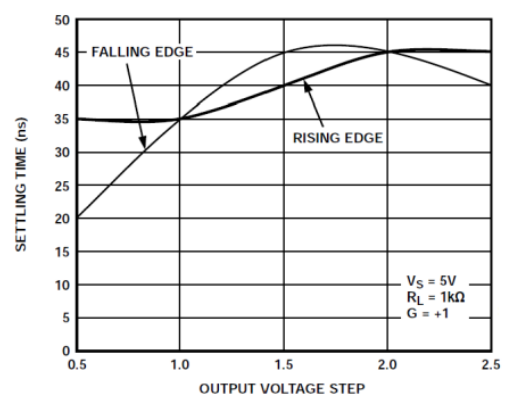

Figure 7. Time vs Output Voltage step

\section{Detect start and stop bits}

The detector monitoring DP bus for real-time, and report the state of the bus. As figure 8 shown, FPGA detect the falling signals of QS, when the bus is idle state (The low level should be duration more than 1/4tbit, otherwise FPGA would filter the signal. The $t_{\mathrm{BIT}}$ is $0.04 \mathrm{us}$ if Profibus DP use $12 \mathrm{M}$ baud rate). 


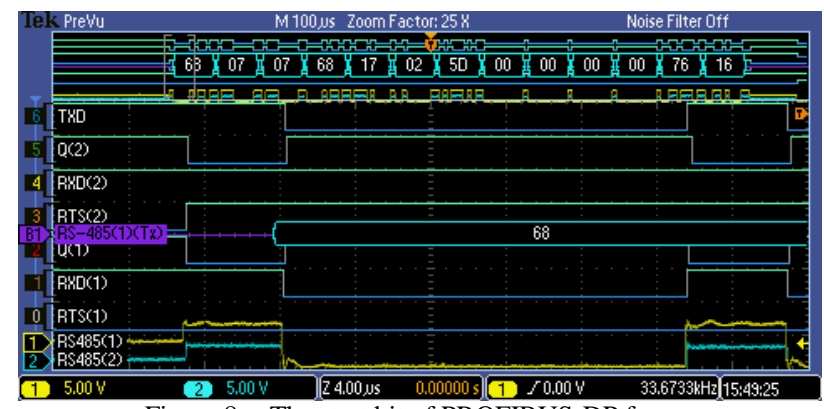

Figure 8. The start bit of PROFIBUS-DP frame

PROFIBUS-DP specified the bus idle time $\mathrm{t}_{\mathrm{SDR}}$ minimum is 11bits which between the last stop bit transmitted over and start bit of next a response message. Taking into account the communication baud rate may up to $12 \mathrm{M}$, so the time is 0.92 us. Therefore, to avoid the bus error, it must be finish the sending and receiving status change of bus transceiver within 0.92 us. As the table 1 shown, the delay time of slave station corresponding to different bus rates. The figure 9 shown, when the QS is a rising edge of any channel, the RXD is high level and duration of less than 11bits, the bus is change into the idle state, the channel transceiver receives message is over.

TABLE 1: THE DELAY TIME OF SLAVE STATION CORRESPONDING TO DIFFERENT BUS RATES

\begin{tabular}{|c|c|c|c|c|c|c|c|}
\hline \multirow{2}{*}{ content } & \multicolumn{7}{|c|}{ kbit*s-1 } \\
\cline { 2 - 8 } & 9.6 & 19.2 & 93.75 & 187.5 & 500 & 1500 & 12000 \\
\hline $\operatorname{minT}_{\text {SDR }}$ & 11 & 11 & 11 & 11 & 11 & 11 & 11 \\
\hline $\max \mathbf{T}_{\text {SDR }}$ & 60 & 60 & 60 & 60 & 100 & 150 & 800 \\
\hline
\end{tabular}

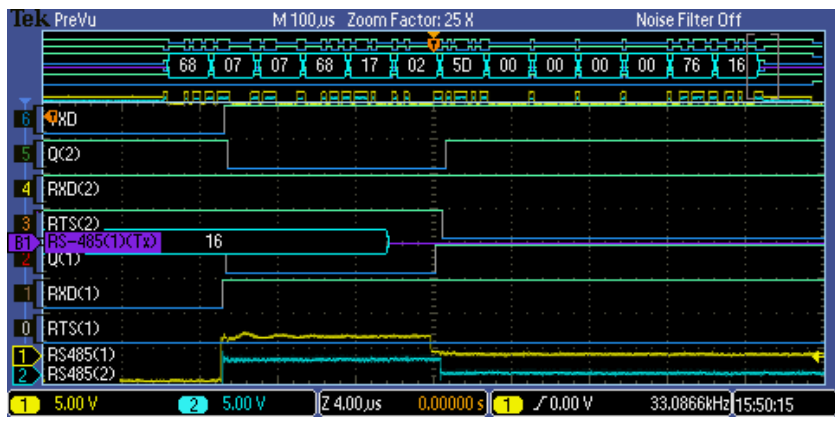

Figure 9. The stop bit of PROFIBUS-DP

DP HUB with $12 \mathrm{M}$ crystal, and $187.5 \mathrm{k}$ baud rates, FPGA process timing and logic state of the full PROFIBUS-DP SD3 frame as shown in Figure 10. In the whole process of network communication, bus transceiver control RTS, receiving and waiting frame RTS is logic low level and forwarding frame RTS is logic high level.

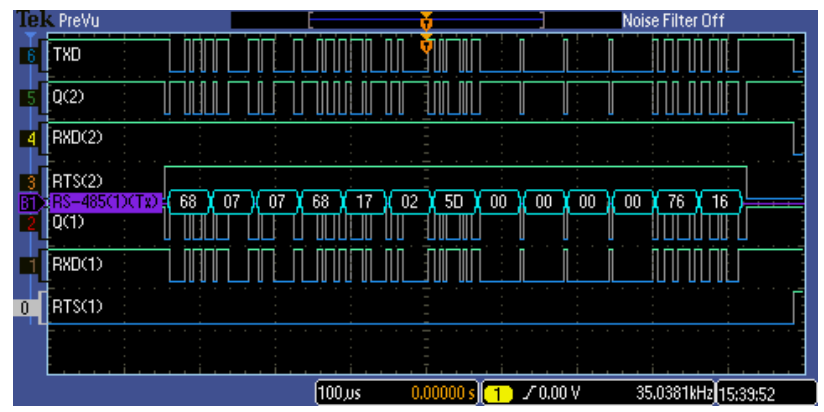

Figure 10. The Idle time of PROFIBUS-DP

\section{CONCLUSIONS}

This paper propose a new design of PROFIBUS-DP HUB, that resolved the past PROFIBUS-DP HUB have common problems of poor universal apply, shape communication is more time-consuming, required message analysis, Drop frame, etc. This design contain a detector which used to monitor the physical layer signal state of the DP bus, use of FPGA synthesis logic signal, could control the direction of data flow in the physical layer, and Unrelated with the upper layer protocol, without message analysis, compliance the protocol which used the standard RS-485, and baudrate-adaptive. Therefore, this new design had some practical value.

\section{ACKNOWLEDGMENT}

This work has been supported by the National High-Tech Research and Development Program of China (Grant No. 2012AA040602-4).

\section{REFERENCES}

[1] Wang Z,Wang T R,Song Y Q,et al. Survey of realtime schedule theory and the state-of-the-are of ITS application in industrial real-time communication system(Part one)[ J], Information and Control,2002,21(2):146-152.)

[2] Fang HE,Weiming TONG Estimation to the Time Parameters of PROFIBUS Network System[J], 2007 Second IEEE Conference on Industrial Electronics and Applications,2007,39(6):873-876.

[3] Siemens SPC3 user description[S],Version : 1.9,2000, http://www.siemens.corn.

[4] Siemens User Description[S],Version : 1.8,1996, http://www.siemens.corn.

[5] Profibus Specification[S],Siemens Corporation,1998 : 36-38.

[6] Profibus specifications[S], Normative parts of Profibus-FMS, Profibus-DP, Profibus-PA according to the European Standard, EN50170,Version 1. 0[Z],1998: 49-138.

[7] Digital data communication for measurement and control Field bus for use in industrial control systems Part 3 : Data link Layer service difinition Type 3(PROFIBUS) IEC61158[s],2003:24-35.

[8] Hein Marais,RS-485Circuit Implementation Guide, http://www.analog.com. 